\title{
Transmission and reflection of Bose-Einstein condensates incident on a Gaussian tunnel barrier
}

\author{
A. M. Martin, ${ }^{1}$ R. G. Scott, ${ }^{2,3}$ and T. M. Fromhold ${ }^{3}$ \\ ${ }^{1}$ School of Physics, University of Melbourne, Parkville, Victoria 3010, Australia \\ ${ }^{2}$ Department of Physics, University of Otago, P.O. Box 56, Dunedin, New Zealand \\ ${ }^{3}$ School of Physics and Astronomy, University of Nottingham, Nottingham NG7 2RD, United Kingdom
}

(Received 2 October 2006; published 28 June 2007)

\begin{abstract}
We investigate how Bose-Einstein condensates, whose initial state is either irrotational or contains a single vortex, scatter off a one-dimensional Gaussian tunnel barrier. We find that for low atom densities the vortex structure within the condensate is maintained during scattering, whereas at medium and high densities, multiple additional vortices can be created by the scattering process, resulting in complex dynamics and disruption of the atom cloud. This disruption originates from two different mechanisms associated, respectively, with the initial rotation of the atom cloud and the interference between the incident and reflected matter waves.
\end{abstract}

DOI: 10.1103/PhysRevA.75.065602

PACS number(s): 03.75.Kk, 05.30.Jp, 67.40.Vs

Recently, several experiments have investigated the scattering of Bose-Einstein condensates (BECs). These experiments have included Bragg reflection in an optical lattice [1], reflection from optical [2] and magnetic [3] mirrors, diffraction from a grating [4], and quantum reflection from a silicon surface [5]. In each case, interest has focused on the reflected component of the BEC. For example, investigations of quantum reflection from a silicon surface have revealed that interatomic interactions have a dramatic effect on the internal structure of the atom cloud $[5,6]$. So far, reflection experiments have been restricted to condensates whose initial state contains no dynamical excitations. However, the methodology for creating and observing vortices in BECs is well established [7-10] and, in the case of Bragg reflection, numerical simulations predict that vortices and solitons in the BEC's initial state strongly influence the subsequent dynamics [11]. Previous theoretical work has shown that the presence of a vortex in the initial state can have a pronounced effect on the internal structure of a BEC that undergoes classical $[12,13]$ or quantum $[14]$ reflection.

In this paper, we investigate how BECs scatter off a Gaussian tunnel barrier, which allows both reflection and quantum-mechanical tunneling of the atoms. We use numerical simulations of the Gross-Pitaevskii equation (GPE) to study how the strength of the interatomic interactions and the vorticity of the initial state affect the scattering properties of the condensate. Our simulations reveal regimes in which dynamical excitations disrupt both the reflected and transmitted atom clouds. In one regime, which we call rotational disruption, the excitations originate from the effect of the initial vortex on the scattering dynamics. By contrast, the regime of interferential disruption [15] occurs both in the presence and absence of a vortex in the initial state, with the excitations being created from interference between the incident and reflected matter waves. Rotational disruption arises when the time taken for the vortex core to scatter is comparable with, or exceeds, its rotational period. Interferential disruption occurs when the scattering time is greater than the correlation time of the BEC.

We consider a BEC containing $N{ }^{23} \mathrm{Na}$ atoms of mass $m$ initially confined by a harmonic trapping potential, $V_{T}(x, y, z)=m\left[\omega_{x}^{2}(x+\Delta x)^{2}+\omega_{y}^{2} y^{2}+\omega_{z} z^{2}\right] / 2 \quad$ centered at
$(-\Delta x, 0,0)$. We consider trap frequencies $\omega_{x}=\omega_{y}=50$ $\times 2 \pi \mathrm{rad} \mathrm{s}^{-1} \ll \omega_{z}$ so that the spatial width of the BEC is much smaller along the $z$ direction than along the $x$ and $y$ directions. Consequently, the dynamics reduce to two-dimensional motion in the $x-y$ plane. At time $t=0$, we create an additional Gaussian tunnel barrier [16], $V_{L}(x)$ $=V_{0} \exp \left(-x^{2} / \sigma^{2}\right)$, of width $\sigma=1 \mu \mathrm{m}$ along the $x$ direction, by switching on a far blue-detuned laser beam that travels along the $y$ direction and creates a sheet of laser light. The intensity of the laser beam determines the barrier height, $V_{0}$, which we take to be $6.2 \mathrm{peV}$, similar to recent experiments [16]. Simultaneously, we accelerate the BEC towards the Gaussian potential by abruptly displacing the harmonic trap through a distance $\Delta x$ along the $x$ direction [4-6]. After the displacement, the center of the trap coincides with the barrier and the total potential energy of the trap and laser beam, for motion in the $x-y$ plane, is given by

$$
V(x, y)=m\left(\omega_{x}^{2} x^{2}+\omega_{y}^{2} y^{2}\right) / 2+V_{L}(x) .
$$

The solid curve in Fig. 1(a) shows the form of $V(x, y=0)$. After the displacement of the harmonic trap, the condensate moves away from its initial state [shown schematically by the dashed curve in Fig. 1(a)], reaches the Gaussian potential (a)

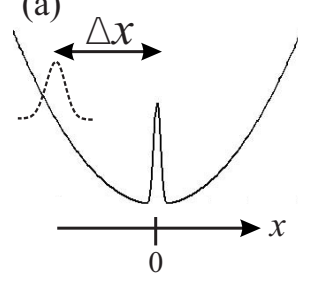

(b)

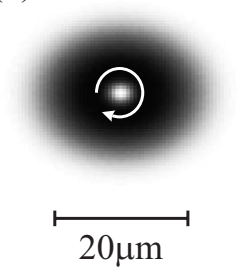

(c)

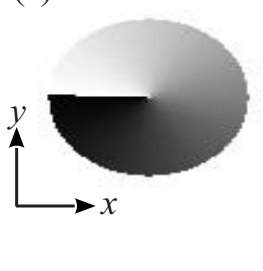

FIG. 1. (a) Solid curve, a schematic of the potential energy $V(x, y=0)$ created by the harmonic trap and laser beam when $t \geqslant 0$; Dashed curve, a schematic of the initial probability density $|\Psi(x, y=0, t=0)|^{2}$ of the initially irrotational BEC $A_{i}$. (b) The initial density $|\Psi(x, y, t=0)|^{2}$ (black represents high density, white represents zero) of BEC $A$, where the white arrow shows the direction of the condensate's circulation and the horizontal bar denotes the scale. (c) The equivalent phase plot, $\phi(x, y, t=0)$ (white represents 0 , black represents $2 \pi$ ). 


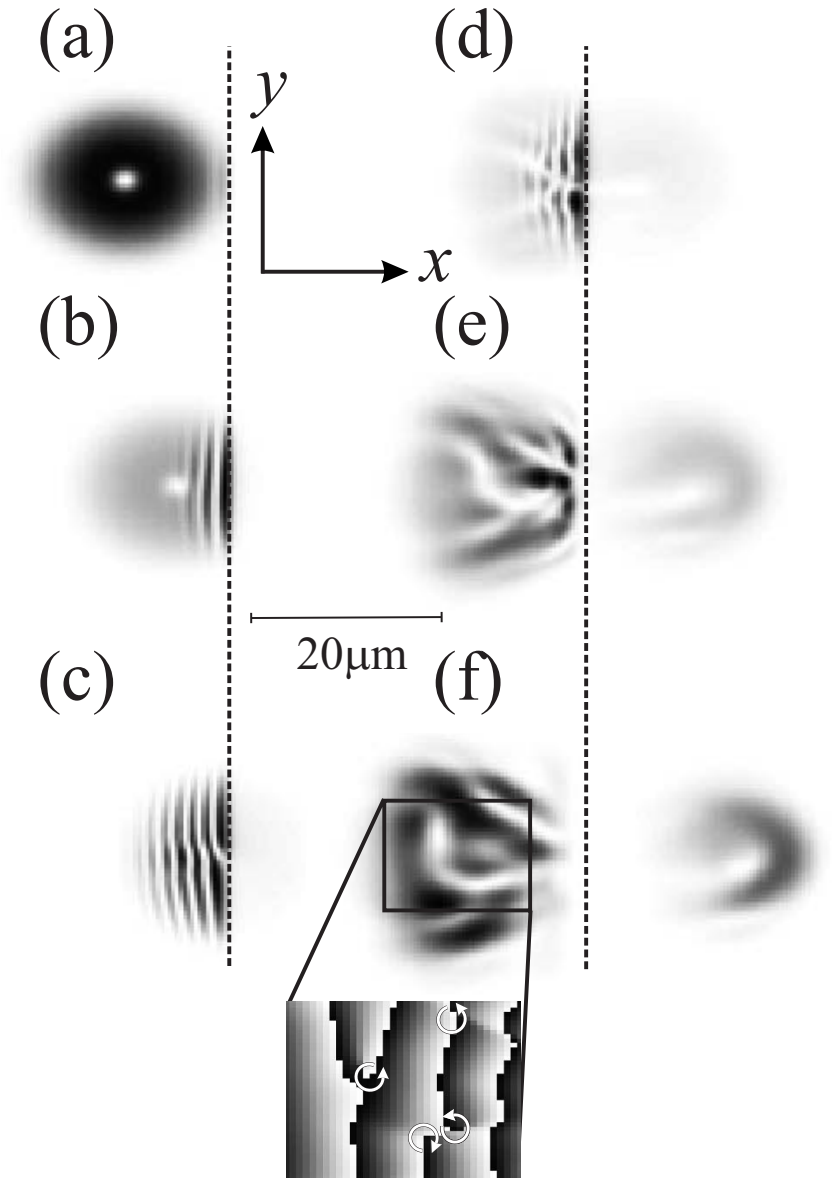

FIG. 2. Evolution of BEC $A$ : plots of $|\Psi(x, y, t)|^{2}$ (black represents high density, white represents zero) for $\bar{v}_{x}=6.3 \mathrm{~mm} \mathrm{~s}^{-1}$ at $t$ $=3 \mathrm{~ms}$ (a), $4 \mathrm{~ms}$ (b), $5 \mathrm{~ms}$ (c), $6 \mathrm{~ms}$ (d), $7 \mathrm{~ms}$ (e), and $8 \mathrm{~ms}$ (f). The dashed line at $x=0$ marks the point where the laser potential is maximal. Coordinate axes are inset and the horizontal bar indicates scale. Lower plot: phase $\phi(x, y, t=8 \mathrm{~ms})$ [white represents 0 , black represents $2 \pi$ ] within the region enclosed by the box in (f). Arrows indicate the direction of circulation.

with a mean incident velocity $\bar{v}_{x} \approx \omega_{x} \Delta x$, and is then scattered by the tunnel barrier.

The time-dependent GPE for the system is

$$
i \hbar \frac{\partial \Psi}{\partial t}=\left[\frac{-\hbar^{2}}{2 m}\left(\frac{\partial^{2}}{\partial x^{2}}+\frac{\partial^{2}}{\partial y^{2}}\right)+V(x, y)+g|\Psi|^{2}\right] \Psi,
$$

where $\Psi(x, y, t)$ is the wave function for motion in the $x-y$ plane at time $t \geqslant 0$ and $g=4 \pi \hbar^{2} a / m$ where the $s$-wave scattering length $a=2.9 \mathrm{~nm}$.

We determine the initial BEC wave function by solving Eq. (2) for $t \leqslant 0$ using an imaginary time algorithm [17]. When the initial state contains a vortex we impose the requirement that there is a $2 \pi$-phase change in the condensate wave function around the trap center at $(x, y)=(-\Delta x, 0)$, which corresponds to a quantized angular momentum of $-\hbar$ about the $z$ axis. The wave function is normalized according to

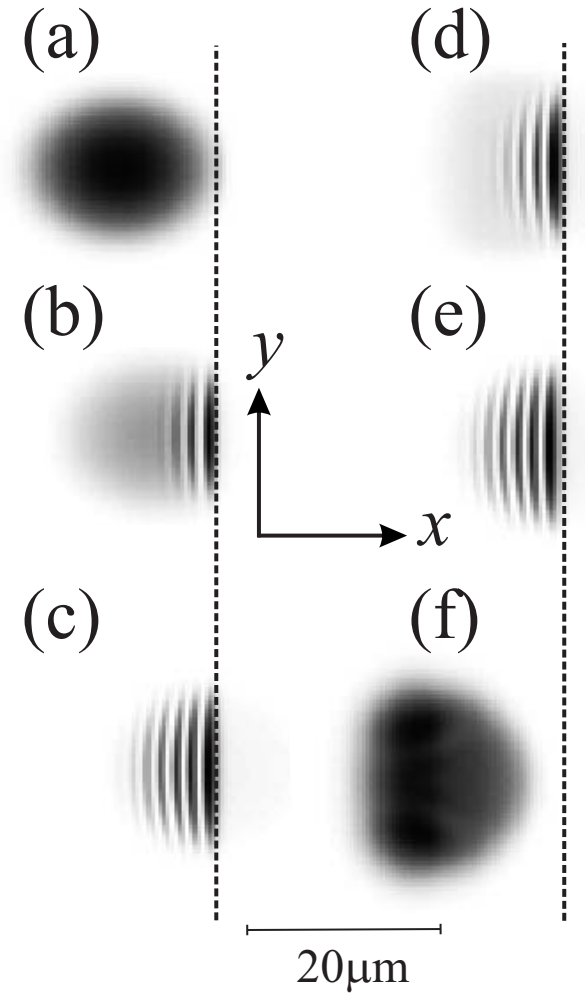

FIG. 3. Evolution of BEC $A_{i}$ : plots of $|\Psi(x, y, t)|^{2}$ (black represents high density, white represents zero) for $\bar{v}_{x}=6.3 \mathrm{~mm} \mathrm{~s}^{-1}$ at $t$ $=3 \mathrm{~ms}$ (a), $4 \mathrm{~ms}$ (b), $5 \mathrm{~ms}$ (c), $6 \mathrm{~ms}$ (d), $7 \mathrm{~ms}$ (e), and $8 \mathrm{~ms}$ (f). The dashed line at $x=0$ marks the point where the laser potential is maximal. Coordinate axes are inset and the horizontal bar indicates scale.

$$
\int|\Psi(x, y, t)|^{2} d x d y=\frac{N}{L_{z}}
$$

where $L_{z}$ is the confinement length in the $z$ direction. We consider two different rotating BECs with $N / L_{z}=5$ $\times 10^{9} \mathrm{~m}^{-1}($ BEC $A)$ and $N / L_{z}=2.5 \times 10^{11} \mathrm{~m}^{-1}($ BEC $B)$, corresponding to peak atom densities of $n_{0}=2.1 \times 10^{19} \mathrm{~m}^{-3}$ and $n_{0}=1.6 \times 10^{20} \mathrm{~m}^{-3}$, respectively. The density, $|\Psi(x, y, t=0)|^{2}$, and phase, $\phi(x, y, t=0)$, of the initial state of BEC $A$ are shown in Figs. 1(b) and 1(c). Having obtained the initial state of the BEC, we determine its motion by solving the GPE numerically using the Crank-Nicolson method [18]. We identify the effect of the initial vortex by comparing the dynamics of BECs $A$ and $B$ with their irrotational counterparts labeled BECs $A_{i}$ and $B_{i}$ respectively.

Figure 2 shows the density profile of BEC $A$ after a trap displacement of $20 \mu \mathrm{m}\left(\bar{v}_{x}=6.3 \mathrm{~mm} \mathrm{~s}^{-1}\right)$. As the BEC impinges upon the Gaussian tunnel barrier, a standing wave forms between the incoming and reflected matter waves. Figure 2(b) shows the first stage of the standing wave formation in which maxima (black) and nodal lines (white) appear at the leading edge of the atom cloud. In Figs. 2(c) and 2(d) the standing wave undergoes a $\pi$ phase shift between the upper and lower edges of the BEC. This is due to the nonuniform initial phase of the BEC, shown in Fig. 1(c). After scattering, the BEC splits into reflected $(x<0)$ and transmitted $(x>0)$ 


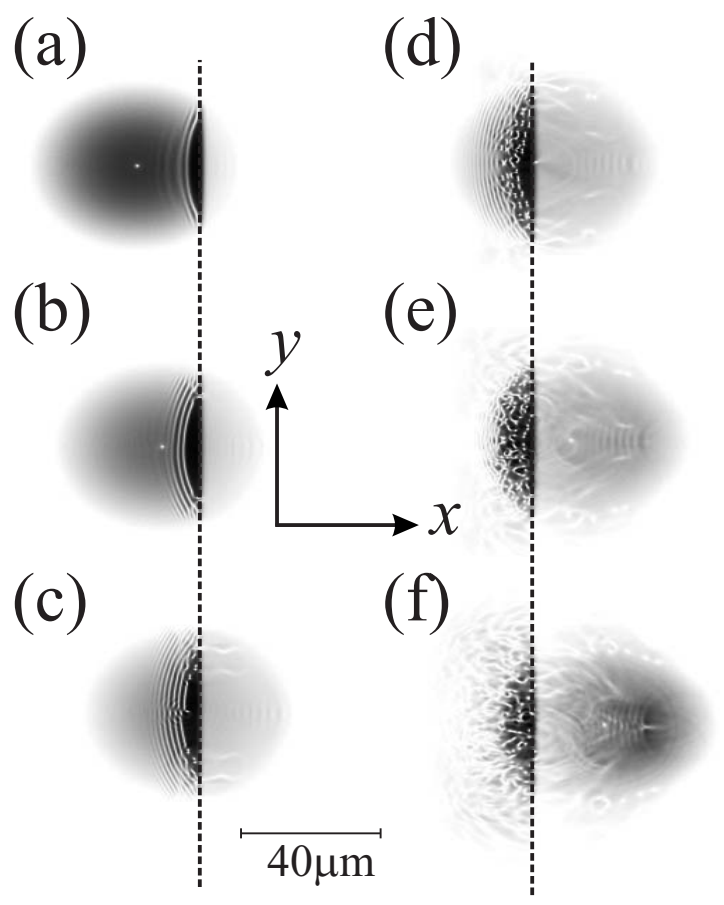

FIG. 4. Evolution of BEC $B$ : plots of $|\Psi(x, y, t)|^{2}$ (black represents high density, white represents zero) for $\bar{v}_{x}=9.4 \mathrm{~mm} \mathrm{~s}^{-1}$ at $3 \mathrm{~ms}$ (a), $4 \mathrm{~ms} \mathrm{(b),} 5 \mathrm{~ms}$ (c), $6 \mathrm{~ms}$ (d), $7 \mathrm{~ms}$ (e), and $8 \mathrm{~ms}$ (f). The dashed line at $x=0$ marks the point where the laser potential is maximal. Coordinate axes are inset and the horizontal bar indicates the scale.

components [Figs. 2(d)-2(f)]. The reflected component of the BEC is significantly disrupted [Figs. 2(e) and 2(f)]. This disruption is accompanied by the formation of new vortices within the boxed region in Fig. 2(f). The phase variation, $\phi(x, y, t=8 \mathrm{~ms})$, within this region is shown in the lower part of Fig. 2(f).

Figure 3 shows density profiles for the initially irrotational BEC $A_{i}$ after a trap displacement of $20 \mu \mathrm{m}$ $\left(\bar{v}_{x}=6.3 \mathrm{~mm} \mathrm{~s}^{-1}\right)$. No dynamical excitations are produced in either the transmitted or reflected clouds. We therefore conclude that the disruption observed for BEC A (Fig. 2) is related to its initial rotation.

The effect of the vortex on the scattering process depends on the scattering time of the vortex core $\left(t_{s v}\right)$ relative to its rotation period $\left(t_{r}\right)$. Since the diameter of the vortex core is approximately the healing length $\xi=1 / \sqrt{8 \pi n_{0} a}$, it follows that $t_{s v} \approx 2 \xi / \bar{v}_{x}$ and $t_{r}=\pi \xi^{2} m / h$. If the ratio

$$
\frac{t_{r}}{t_{s v}}=\frac{m}{2 h} \pi \xi \Delta x \omega_{x} \propto \frac{\Delta x}{\sqrt{n_{0}}}
$$

is $\gg 1$, the vortex will have little effect on the dynamics of the transmitted and reflected atom clouds. This occurs for low density BECs in which the spread of incident velocities, produced by the rotation, is small compared to $\bar{v}_{x}$. Conversely, if $t_{r} / t_{s v} \lesssim 1$, as is the case for BEC $A$, the rotation of the BEC will disrupt the reflected atom cloud (rotational disruption), as shown in Fig. 2.

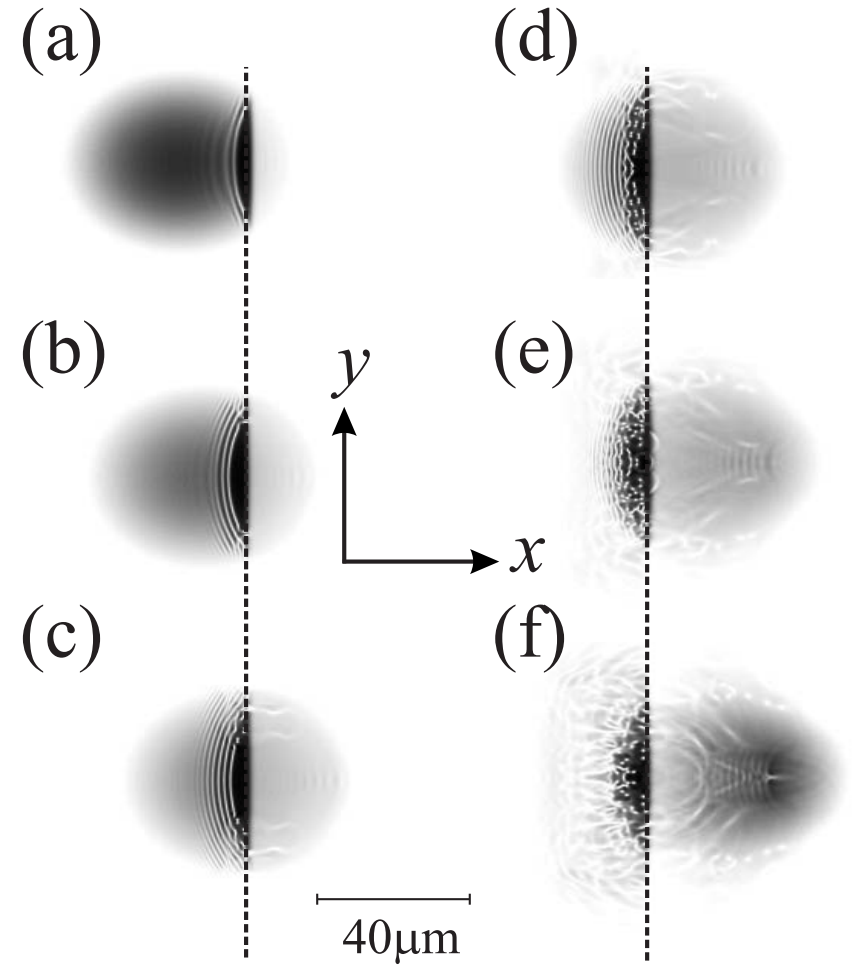

FIG. 5. Evolution of BEC $B_{i}$ : plots of $|\Psi(x, y, t)|^{2}$ (black represents high density, white represents zero) for $\bar{v}_{x}=9.4 \mathrm{~mm} \mathrm{~s}^{-1}$ at $t$ $=3 \mathrm{~ms}$ (a), $4 \mathrm{~ms} \mathrm{(b),} 5 \mathrm{~ms}$ (c), $6 \mathrm{~ms}$ (d), $7 \mathrm{~ms}$ (e), and $8 \mathrm{~ms}$ (f). The dashed line at $x=0$ marks the point where the laser potential is maximal. Coordinate axes are inset and the horizontal bar indicates the scale.

Disruption of the reflected cloud is even more pronounced for the higher density BEC $B$. Fig. 4 shows the density of this BEC after a trap displacement of $30 \mu \mathrm{m}$ $\left(\bar{v}_{x}=9.4 \mathrm{~mm} \mathrm{~s}^{-1}\right)$. As the condensate impinges upon the scattering potential, a standing wave forms between the incident and reflected matter waves, Figs. 4(a) and 4(b). This standing wave seeds solitons $[6,19,20]$, which decay via the snake instability [21] into vortex-antivortex pairs [Figs. 4(c) and 4(d)], thus strongly disrupting the internal structure of the cloud [Figs. 4(e) and 4(f)]. Consequently, when incident atoms subsequently pass through the barrier they produce some irregularity in the transmitted atom cloud. Note that this irregularity is less pronounced towards the right-hand edge of the transmitted cloud, which contains the atoms that passed through the barrier before solitons and vortices formed at negative $x$. We emphasize that although BEC $B$ is in a regime where rotational disruption occurs, the initial vortex does not (in contrast to BEC A) cause the severe fragmentation shown in Fig. 4. To demonstrate this, Fig. 5 shows the initially irrotational BEC $B_{i}$ scattering off a Gaussian potential after a trap displacement of $30 \mu \mathrm{m}$ $\left(\bar{v}_{x}=9.4 \mathrm{~mm} \mathrm{~s}^{-1}\right)$. Comparison of Figs. 4 and 5 reveals that the dynamics are qualitatively the same irrespective of whether or not the BEC contains an initial vortex.

To understand these results, we recall previous work $[11,19,20,22]$ on the Bragg reflection of a BEC in an optical lattice. In Refs. $[19,20]$ it was shown that at Bragg reflection, fragmentation can arise from the density and phase imprint- 
ing that accompanies standing wave formation. When the correlation time, $t_{c}=m /\left(2 \sqrt{2} h n_{0} a\right)$, is much less than the Bloch period $\left(t_{B}\right)$, this imprinting leads to the formation of solitons and vortices, which disrupt the atom cloud. For BECs $B$ and $B_{i}$, a similar disruption occurs when $t_{c} \ll t_{s}$, where $t_{s}=l_{x} / \bar{v}_{x}$ is the approximate duration of the reflection process. We call this effect interferential disruption [15], since it originates from density fluctuations across an interference pattern, in this case produced by the superposition of the incident and reflected matter waves. For BEC $B$ this interferential disruption dominates the dynamics and completely masks any effects due to the rotational disruption.

We have investigated how BECs with different atom densities scatter off a Gaussian tunnel barrier when the initial state is either irrotational or contains a single vortex. We find three distinct dynamical regimes, which occur for different atom densities: (i) at low densities $\left(\leq 10^{19} \mathrm{~m}^{-3}\right)$ there is no fragmentation of the reflected or transmitted components of the BEC, irrespective of whether or not there is a vortex in the initial state; (ii) at medium densities $\left(10^{19}-10^{20} \mathrm{~m}^{-3}\right)$ for which $t_{r} / t_{s r} \lesssim 1$, rotational disruption occurs in the reflected component of a BEC with an initial vortex, but no disruption is observed when the BEC is initially irrotational; (iii) at high densities $\left(\gtrsim 10^{20} \mathrm{~m}^{-3}\right)$, there is strong interferential disruption in the reflected atom cloud if $t_{c} \lesssim t_{s}$, both in the presence and absence of an initial vortex.

This work was supported by the ARC, EPSRC UK, the Royal Society (London) and the University of Melbourne.
[1] O. Morsch, J. H. Müller, M. Cristiani, D. Ciampini, and E. Arimondo, Phys. Rev. Lett. 87, 140402 (2001).

[2] A. S. Arnold, C. MacCormick, and M. G. Boshier, Phys. Rev. A 65, 031601(R) (2002).

[3] K. Bongs, S. Burger, G. Birkl, K. Sengstock, W. Ertmer, K. Rzazewski, A. Sanpera, and M. Lewenstein, Phys. Rev. Lett. 83, 3577 (1999).

[4] A. Günther, S. Kraft, M. Kemmler, D. Koelle, R. Kleiner, C. Zimmermann, and J. Fortágh, Phys. Rev. Lett. 95, 170405 (2005).

[5] T. A. Pasquini, Y. Shin, C. Sanner, M. Saba, A. Schirotzek, D. E. Pritchard, and W. Ketterle, Phys. Rev. Lett. 93, 223201 (2004); T. A. Pasquini, M. Saba, G.-B. Jo, Y. Shin, W. Ketterle, D. E. Pritchard, T. A. Savas, and N. Mulders, ibid. 97, 093201 (2006)

[6] R. G. Scott, A. M. Martin, T. M. Fromhold, and F. W. Sheard, Phys. Rev. Lett. 95, 073201 (2005).

[7] M. R. Matthews, B. P. Anderson, P. C. Haljan, D. S. Hall, C. E. Wieman, and E. A. Cornell, Phys. Rev. Lett. 83, 2498 (1999).

[8] K. W. Madison, F. Chevy, W. Wohlleben, and J. Dalibard, Phys. Rev. Lett. 84, 806 (2000).

[9] F. Chevy, K. W. Madison, and J. Dalibard, Phys. Rev. Lett. 85, 2223 (2000).

[10] C. Raman, J. R. Abo-Shaeer, J. M. Vogels, K. Xu, and W. Ketterle, Phys. Rev. Lett. 87, 210402 (2001).

[11] R. G. Scott, A. M. Martin, and T. M. Fromhold, Phys. Rev. A
69, 063607 (2004).

[12] J. J. Garcia-Ripoll, G. Molina-Terriza, V. M. Perez-Garcia, and L. Torner, Phys. Rev. Lett. 87, 140403 (2001).

[13] I. Josopait, L. Dobrek, L. Santos, A. Sanpera, and M. Lewenstein, Eur. Phys. J. D 22, 385 (2003).

[14] M. Liu, L. She, H. W. Xiong, and M. S. Zhan, Phys. Rev. A 74, 043619 (2006).

[15] R. G. Scott, D. A. W. Hutchinson, and C. W. Gardiner, Phys. Rev. A 74, 053605 (2006).

[16] Y. Shin, M. Saba, T. A. Pasquini, W. Ketterle, D. E. Pritchard, and A. E. Leanhardt, Phys. Rev. Lett. 92, 050405 (2004).

[17] M. L. Chiofalo, S. Succi, and M. P. Tosi, Phys. Rev. E 62, 7438 (2000).

[18] W. H. Press, S. A. Teukolsky, W. T. Vetterling, and B. P. Flannery, Numerical Recipes, the Art of Scientific Computing (Cambridge University Press, Cambridge, 1994).

[19] R. G. Scott, A. M. Martin, S. Bujkiewicz, T. M. Fromhold, N. Malossi, O. Morsch, M. Cristiani, and E. Arimondo, Phys. Rev. A 69, 033605 (2004).

[20] R. G. Scott, A. M. Martin, T. M. Fromhold, S. Bujkiewicz, F. W. Sheard, and M. Leadbeater, Phys. Rev. Lett. 90, 110404 (2003).

[21] Z. Dutton, M. Budde, C. Slowe, and L. V. Hau, Science 293, 663 (2001).

[22] L. Fallani, L. De Sarlo, J. E. Lye, M. Modugno, R. Saers, C. Fort, and M. Inguscio, Phys. Rev. Lett. 93, 140406 (2004). 